\title{
Sound and Complete Monitoring of Sequential Consistency for Relaxed Memory Models
}

\author{
Jabob Burnim, Koushik Sen, and Christos Stergiou \\ EECS Department, University of California, Berkeley \\ \{jburnim,ksen, chster\}@cs.berkeley.edu
}

\begin{abstract}
We present a technique for verifying that a program has no executions violating sequential consistency (SC) when run under the relaxed memory models Total Store Order (TSO) and Partial Store Order (PSO). The technique works by monitoring sequentially consistent executions of a program to detect if similar program executions could fail to be sequentially consistent under TSO or PSO. We propose novel monitoring algorithms that are sound and complete for TSO and PSO - if a program can exhibit an SC violation under TSO or PSO, then the corresponding monitor can detect this on some SC execution. The monitoring algorithms arise naturally from the operational definitions of these relaxed memory models, highlighting an advantage of viewing relaxed memory models operationally rather than axiomatically. We apply our technique to several concurrent data structures and synchronization primitives, detecting a number of violations of sequential consistency.
\end{abstract}

\section{Introduction}

Programmers writing concurrent software often assume that the underlying memory model is sequentially consistent. However, sequential consistency strongly constrains the ordering of memory operations, which can make it difficult to achieve high performance in commodity microprocessors 920 . Thus, to enable increased concurrency and performance, processors often provide a relaxed memory model. Unfortunately, working with relaxed memory models often requires subtle and difficult reasoning 920].

Nevertheless, developers of high-performance concurrent programs, such as lock-free data-structures and synchronization libraries, often use regular load and store operations, atomic compare-and-swap-like primitives, and explicit data races instead of locks to increase performance. Concurrency bugs are notoriously hard to detect and debug; relaxed memory models make the situation even worse.

Recently, there has been great interest in developing techniques for the verification and analysis of concurrent programs under relaxed memory mod-

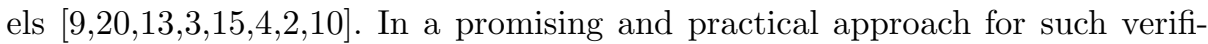
cation, Burckhardt and Musuvathi [4] argued that programmers, despite using ad-hoc synchronization, expect their program to be sequentially consistent. They proposed SOBER, which monitors sequentially consistent executions to detect violations of sequential consistency (SC). A key observation made in their work

P.A. Abdulla and K.R.M. Leino (Eds.): TACAS 2011, LNCS 6605, pp. 11-25, 2011.

(C) Springer-Verlag Berlin Heidelberg 2011 
is that, for the Total Store Order (TSO) 23] memory model (which is quite similar to that of the $\mathrm{x} 86$ architecture 21]), if a program execution under TSO violates sequential consistency (SC), then this fact can be detected by examining some sequentially consistent execution of the program. Therefore, if run-time monitoring is combined with a traditional model checker, which explores all sequentially consistent executions of the program, then all violations of SC under TSO can be detected. Burckhardt and Musuvathi 4 use an axiomatic definition of SC and TSO to derive the SOBER monitoring algorithm.

In this paper, we develop two novel monitoring algorithms for detecting violations of sequential consistency (SC) under relaxed memory models Total Store Order (TSO) 23] and Partial Store Order (PSO) 23. Each algorithm, when monitoring a sequentially consistent execution of a program, simulates a similar TSO or PSO execution, reporting if this similar execution can ever violate sequential consistency. We prove both monitors sound - if they report a warning, then the monitored program can violate SC under TSO or PSO - and completeif a program can violate $\mathrm{SC}$ under $\mathrm{TSO}$ or $\mathrm{PSO}$, then the corresponding monitor can detect this fact by examining some sequentially consistent execution.

Rather than working with axiomatic definitions of these relaxed memory models, as 4 does, we derive our algorithms from operational definitions of TSO and PSO. We show that this alternate approach naturally leads to fundamentally different monitoring algorithms, with several advantages over SOBER.

One advantage of our operational approach is that our monitoring algorithms follow simply from the operational definitions of TSO and PSO. While monitoring algorithms based on axiomatic definitions require the design of complex vector clocks, in addition to the standard vector clocks to track the traditional happens-before relation, our approach can directly "run" the operational memory model. Thus, we were easily able to develop a monitoring algorithm for PSO, in addition to TSO - such an extension is unknown for the SOBER algorithm.

Another advantage of our algorithms is that they have a run-time complexity of $O(N \cdot P)$ when monitoring a sequentially consistent execution with $N$ shared memory operations and $P$ processes. This complexity is an improvement over the run-time complexity $O\left(N \cdot P^{2}\right)$ of the SOBER algorithm. We see this improvement in run-time complexity because we do not need to maintain additional vector clocks for TSO and PSO.

Further, in developing our monitoring algorithms, we discovered a bug in the SOBER algorithm - in its axiomatic definition of TSO - which makes the algorithm incomplete. We believe that this bug is quite subtle. The same bug is also present 21] in the 2007 Intel@ 64 Architecture Memory Order White Paper. In this paper, we identify and correct the error [8] in the SOBER algorithm arising from a too-strict axiomatic definition of the TSO memory model.

We have implemented our monitoring algorithms for $\mathrm{C}$ programs in THRILLE [16] and, combined with THRILLE's preemption-bounded model checking [19], have applied our monitors to two mutual exclusion algorithms and several concurrent data structures. Our experiments show that we can detect sequential consistency violations under TSO or PSO in all of these benchmarks. 
Other Related Work. There have been many efforts to verify or check concurrent programs on relaxed memory models 920133154210. Some of these techniques [13.3] encode a program and the underlying memory model as a constraint system and use a constraint solver to find bugs. Other techniques 92015 explicitly explore the state space of a program to find bugs.

Recently, 10 proposed an adversarial memory for testing at run-time if a data race in a Java program could be harmful under Java's memory model.

\section{Preliminaries}

We consider a parallel program $P$ to consist of a number of parallel threads, each with a thread-local state, that communicate though a shared memory. The execution of a parallel program consists of a sequence of program steps. In each step, one of the program threads issues a shared memory operation-e.g., a read or write of a shared variable - and then updates its local state based on its current local state and anything returned by the memory operation-e.g., the value read for a shared variable.

Below, we will give operational definitions of relaxed memory models TSO and PSO as abstract machines. These abstract machine definitions are designed to be simple for a programmer to understand and to reason about, not to exactly describe the internal structure or operation of real hardware processors. For example, our operational definitions contain no caches or mechanisms for ensuring cache coherency. At the same time, these operational definitions are faithful in that they allow exactly the program behaviors allowed by more traditional axiomatic definitions of TSO and PSO.

\subsection{Programming Model}

Let Proc be set of all program processes or thread identifiers and Value be the set of possible program values. Then, we define the set Event of shared memory operations, or events, to consist of all:

- Stores $\mathbf{s t}(p, a, v)$ by process $p \in$ Proc to address $a \in A d r$ of $v \in$ Value.

- Loads $\mathbf{l d}(p, a)$ by process $p \in$ Proc of address $a \in A d r$.

- Atomic operations $\operatorname{atm}(p, a, f)$ by $p \in$ Proc on $a \in A d r$, which store $f(v)$ to address $a$ after reading $v \in$ Value from $a$, where $f:$ Value $\rightarrow$ Value.

This operations models atomic shared memory primitives such as compareand-swap (CAS), fetch-and-add, test-and-set, etc 1

Note that we need not explicitly include a memory fence operation. In our operational models, memory barriers can be simulated by atomic operations $\operatorname{atm}(p, a, f)$, which restrict reordering or delaying of earlier memory ops.

${ }^{1}$ For example, a $\operatorname{CAS}(a$, old, new $)$ by process $p$ is modeled by $\operatorname{atm}(p, a, f)$, where $f=(\lambda x$. if $x=$ old then new else $x)$. The CAS "succeeds" when it reads value old, and "fails" otherwise. 
We denote the process $p$ and address $a$ of an event $e \in$ Event by $p(e)$ and $a(e)$, respectively.

We now formalize programs as independent processes with local state and communicating only via the above shared memory operations. We abstract unnecessary details such as the source language, control flow, and structure of the process-local state. We define a program $P$ to be a tuple $\left(s_{0}\right.$, next, update) where:

- Function $s_{0}:$ Proc $\rightarrow \Sigma$ is the initial program state, mapping process $p$ to its thread-local state $s_{0}(p) \in \Sigma$, where $\Sigma$ is the set of all possible local states.

- Partial function next: Proc $\times \Sigma \rightarrow$ Event indicates the next memory operation or event, next $(p, \sigma)$, that process $p$ issues when in local state $\sigma$.

If next is undefined for $(p, \sigma)$, denoted $n \operatorname{ext}(p, \sigma)=\perp$, then process $p$ is complete. Program $P$ terminates when $\operatorname{next}(p, s(p))=\perp$ for all $p$.

- Function update : Proc $\times \Sigma \times$ Value $\rightarrow \Sigma$ indicates the new local state update $(p, \sigma, v)$ of process $p$ after it receives response $v$ to op $\operatorname{next}(p, \sigma)$.

We similarly formalize a memory model $M M$ as a labeled transition system with initial state $m_{0}$ and with transitions labeled by events from Event, paired with memory model responses. We also allow memory model $M M$ to have a set of labeled internal transitions $\tau_{M M}$, to model any internal nondeterminism of the memory model. Then, an execution of program $P$ under memory model $M M$ is a sequence of labeled transitions:

$$
\left(s_{0}, m_{0}\right) \stackrel{l_{1}}{\longrightarrow}\left(s_{1}, m_{1}\right) \stackrel{l_{2}}{\longrightarrow} \cdots \stackrel{l_{n}}{\longrightarrow}\left(s_{n}, m_{n}\right)
$$

where each transition $\left(s_{i-1}, m_{i-1}\right) \stackrel{l_{i}}{\longrightarrow}\left(s_{i}, m_{i}\right)$ is either labeled by an ordered pair $\left(e_{i}, r_{i}\right) \in$ Event $\times$ Value, in which case:

$-e_{i}=\operatorname{next}\left(p\left(e_{i}\right), s_{i-1}\left(p\left(e_{i}\right)\right)\right)$

$-s_{i}\left(p\left(e_{i}\right)\right)=$ update $\left(p\left(e_{i}\right), s_{i-1}\left(p\left(e_{i}\right)\right), r_{i}\right)$, where $r_{i}$ is the value returned for $e_{i}$, which is $\perp$ for stores.

$-s_{i}\left(p^{\prime}\right)=s_{i-1}\left(p^{\prime}\right)$ for $p^{\prime} \neq p\left(e_{i}\right)$

- $m_{i-1} \stackrel{\left(e_{i}, r_{i}\right)}{\longrightarrow} m_{i}$ is a transition in $M M$

or is labeled by an internal transition from $\tau_{M M}$, in which case:

$-s_{i}=s_{i-1}$, and $m_{i-1} \stackrel{l_{i}}{\longrightarrow} m_{i}$ is a transition in $M M$

In this model, there are two sources of nondeterminism in a program execution: (1) The thread schedule - i.e., at each step, which process $p$ executes a transition, and (2) the internal nondeterminism of the memory model.

\section{Operational Memory Models}

We now give our operational definitions for three memory models: sequential consistency (SC) and relaxed memory models TSO and PSO. Fundamentally, these definitions are equivalent to operational definitions given by other researchers for SC [145218], TSO [14522118], and PSO [14218]. 
In our presentation, we aim for definitions that provide a simple and easy to understand model for a programmer. We present each memory model as a library or module with an internal state, representing the abstract state of a shared memory, and with methods $\operatorname{store}(p, a, v), \operatorname{load}(p, a)$, and atomic $(p, a, f)$ by which a program interacts with the shared memory. The memory model executes all such methods atomically - i.e. one at a time and uninterrupted. Additionally, memory models TSO and PSO each have an internal method store ${ }^{c}$. Each memory model is permitted to nondeterministically call this internal method, with any arguments, whenever no other memory model method is being executed.

A note about connecting these definitions to our formalism in Section 2,

- $m \stackrel{\operatorname{ld}(p, a), v}{\longrightarrow} m$ when $\operatorname{load}(p, a)$, run in memory model state $m$, returns $v$. (Note that $\operatorname{load}(p, a)$ does not modify the memory model state $m$.)

- $m \stackrel{\text { st }(p, a, v), \perp}{\longrightarrow} m^{\prime}$ when $\operatorname{store}(p, a, v)$, run in state $m$, yields state $m^{\prime}$.

- $m \stackrel{\operatorname{atm}(p, a, f), v}{\longrightarrow} m^{\prime}$ when atomic $(p, a, f)$, run in $m$, returns $v$ and yields $m^{\prime}$.

Sequential Consistency (SC). Our operational definition of SC [17] is given in Figure 1. The SC abstract machine simply models shared memory as an array $m$ mapping addresses to values, reading from and writing to this array on loads, stores, and atomic operations.

Total Store Order (TSO). Our definition of TSO 23] is given in Figure 2 In addition to modeling shared memory as array $m$, mapping addresses to values, the TSO abstract machine has a FIFO write buffer $B[p]$ for each process $p$.

We omit a proof that our operational definition is equivalent to more traditional axiomatic ones. Our model is similar to the operational definitions in [4] and 21], both of which are proved equivalent to axiomatic definitions of TSO. Conceptually, the per-process write buffers allow stores to be reordered or delayed past later loads, while ensuring that each process's stores become globally visible in the order in which they are performed. And there is a total order on all stores - the order in which stores commit - that respects the program order.

Partial Store Order (PSO). Our operational definition of PSO [23] is given in Figure 3, Our PSO abstract machine is very similar to that of TSO, except that pending writes are stored in per-process and per-address write buffers. Internal method $\operatorname{store}_{P S O}^{c}(p, a)$ commits the oldest pending store to address $a$ and $\operatorname{atomic}_{P S O}(p, a, f)$ commits/flushes only pending stores to address a.

\begin{tabular}{|c|c|c|}
\hline $\mathrm{m}[A d r]:$ Val & & $\begin{array}{c}\operatorname{atomic}_{\mathrm{SC}}(p, a, f): \\
\text { ret }:=m[a]\end{array}$ \\
\hline $\begin{array}{c}\operatorname{store}_{\mathrm{SC}}(p, a, v) \\
m[a]:=v\end{array}$ & $\begin{array}{l}\operatorname{load}_{\mathrm{SC}}(p, a): \\
\quad \operatorname{return} m[a]\end{array}$ & $\begin{array}{l}m[a]:=f(m[a]) \\
\text { return ret }\end{array}$ \\
\hline
\end{tabular}

Fig. 1. Operational Model of Sequential Consistency (SC) 


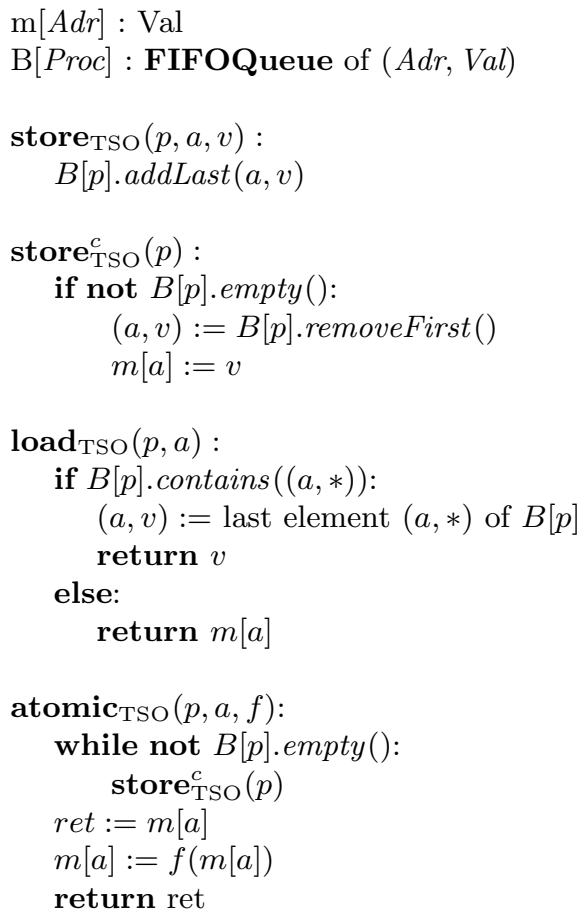

Fig. 2. Operational Model of TSO

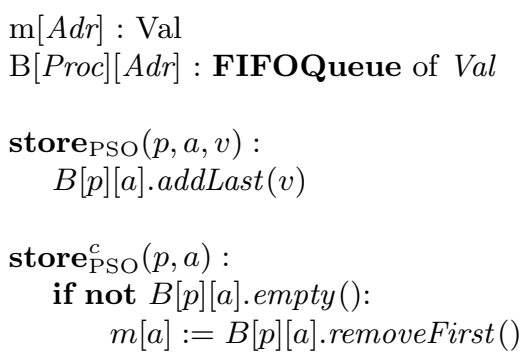

else:

return $m[a]$

$\operatorname{atomic}_{\mathrm{PSO}}(p, a, f)$ :

while not $B[p][a]$.empty () :

$\operatorname{store}_{\mathrm{PSO}}^{c}(p, a)$

ret $:=m[a]$

$m[a]:=f(m[a])$

return ret

Fig. 3. Operational Model of PSO

\section{Violations of Sequential Consistency}

In this section, we formally define what it means for a program to have a violation of sequential consistency under a relaxed memory model. In Section 5, we will give monitoring algorithms for detecting such violations under TSO and PSO by examining only the SC executions of a program.

\subsection{Execution Traces}

If a program exhibits some behavior in a TSO or PSO execution, we would like to say that the behavior is not sequentially consistent if there is no execution under SC exhibiting the same behavior. We will define a trace of an SC, TSO, or PSO execution to capture this notion of the behavior of a program execution.

Following [12], 4], and [6], we formally define a trace of an execution of a program $P$ to be a tuple $\left(E, \rightarrow_{p}, s r c, \rightarrow_{s t}\right)$, where

- $E \subseteq$ Event is the set of shared memory operations or events in the execution.

- For each process $p \in$ Proc, relation $\rightarrow p \subseteq E \times E$ is a total order on the events $e \in E$ from process $p$-i.e. with $p(e)=p$. In particular, $e \rightarrow p$ iff $p(e)=p\left(e^{\prime}\right)$ and $e$ is before $e^{\prime}$ in the execution. Thus, $\rightarrow p$ does not relate events from different processes. 
Relation $\rightarrow_{p}$ is called the program order relation, and $e \rightarrow_{p} e^{\prime}$ indicates that process $p$ issued operation $e$ before issuing $e^{\prime}$.

- For each load or atomic operation $e \in E$, partial function src : Event $\rightarrow$ Event indicates the store or atomic operation $\operatorname{src}(e) \in$ Event from which $e$ reads its value. $\operatorname{src}(e)=\perp$ indicates that $e$ got its value from no store, instead reading the initial default value in the shared memory. Note that $a(\operatorname{src}(e))=a(e)$ whenever $\operatorname{src}(e)$ is defined.

- For each address $a$, relation $\rightarrow s t \subseteq E \times E$ is a total order on the stores and atomic operations on $a$ in the execution. In particular, $e \rightarrow_{s t} e^{\prime}$ iff $a(e)=a\left(e^{\prime}\right)$ and $e$ becomes globally visible before $e^{\prime}$ in the execution. Thus, $\rightarrow$ st does not relate events on different addresses.

Memory models SC, TSO, and PSO all guarantee the existence, for each address, of such a total order in which the writes to that address become globally visible 2 . Note that not all relaxed memory models guarantee the existence of such an ordering.

Note that we can only define a trace for a complete TSO or PSO execution - that is, one in which every store has become globally visible or, in the language of our abstract models, committed. If multiple processes have pending writes to the same address, then the execution does not specify an order on those writes and thus does not define a total $\rightarrow$ st relation.

\subsection{Sequential Consistency and the Happens-Before Relation}

We define a trace of a program $P$ to be sequentially consistent only if it arises from some sequentially consistent execution of $P$ :

Definition 1. A trace $T=\left(E, \rightarrow p\right.$, src, $\left.\rightarrow{ }_{s t}\right)$ of a program $P$ is sequentially consistent iff there exists some execution of $P$ under $S C$ with trace $T$.

This definition is not very convenient for showing that a trace is not sequentially consistent. Thus, following 224, we give an axiomatic characterization of SC traces by defining relations $\rightarrow_{c}$ and $\rightarrow_{h b}$ on the events of a trace:

Definition 2. Let $\left(E, \rightarrow_{p}\right.$, src, $\left.\rightarrow{ }_{s t}\right)$ be a trace. Events $e, e^{\prime} \in E$ are related by the conflict-order relation, denoted $e \rightarrow_{c} e^{\prime}$, iff $a(e)=a\left(e^{\prime}\right)$ and one of the following holds:

$-e$ is a write, $e^{\prime}$ is a read, and $e=\operatorname{src}\left(e^{\prime}\right)$,

$-e$ is a write, $e^{\prime}$ is a write, and $e \rightarrow s t e^{\prime}$

$-e$ is a read, $e^{\prime}$ is a write, and either $\operatorname{src}(e)=\perp$ or $\operatorname{src}(e) \rightarrow{ }_{s t} e^{\prime}$

Definition 3. For a trace $\left(E, \rightarrow_{p}\right.$, src, $\left.\rightarrow s t\right)$, the happens-before relation is defined as the union of the program-order and conflict-order relations on $E$-i.e. $\rightarrow_{h b}=\left(\rightarrow_{p} \cup \rightarrow_{c}\right)$. We refer to the reflexive transitive closure of the happensbefore relation as $\rightarrow_{h b}^{*}$.

${ }^{2}$ This property is closely related to store atomicity [1. TSO and PSO do not technically have store atomicity, however, because a process's loads can see the values of the process's earlier stores before those stores become globally visible. 
As observed in [22] and [4, a trace is sequentially consistent iff its $\rightarrow h b$ relation is acyclic:

Proposition 1. Let $T=\left(E, \rightarrow_{p}\right.$, src,$\left.\rightarrow_{s t}\right)$ be a trace of an execution of program $P$. Trace $T$ is sequentially consistent iff relation $\rightarrow_{h b}$ is acyclic on $E$.

We define a sequential consistency violation as a program execution with a nonsequentially-consistent trace:

Definition 4. A program $P$ has a violation of sequential consistency under relaxed memory model TSO (resp. PSO) iff there exists some TSO (resp. PSO) execution of $P$ with trace $T$ such that $T$ is not sequentially consistent.

\section{Monitoring Algorithms}

We describe our monitoring algorithms for TSO and PSO in this section. We suppose here that we are already using a model checker to explore and to verify the correctness of all sequentially consistent executions of program $P$. A number of existing model checkers [1916] explore the sequentially consistent, interleaved schedules of a parallel program.

Figures 4 and 5 list our monitor algorithms for TSO and PSO. We present our algorithms as online monitors - that is, for an SC execution $\left(s_{0}, m_{0}\right) \stackrel{e_{1}, r_{1}}{\longrightarrow}$ $\ldots \stackrel{e_{n}, r_{n}}{\longrightarrow}\left(s_{n}, m_{n}\right)$ of some program $P$, we run monitor $_{T S O}$ (respectively monitor $\left._{P S O}\right)$ on the execution by:

- Initializing the internal state $B$ and last as described in Figure 4 (resp. 51).

- Then, for each step $e_{i} \in$ Event in the execution, from $e_{1}$ to $e_{n}$, we call $\operatorname{monitor}_{T S O}\left(e_{i}\right)$.

- If any of these calls signals "SC Violation", then $P$ has a sequential consistency violation under TSO (resp., PSO). Note that we do not stop our monitoring algorithm after detecting the first violation. Thus, we find all such violations of sequential consistency along each SC execution.

Conceptually, each monitor algorithm works by simulating a TSO (respectively PSO) version of the given $\mathrm{SC}$ execution. Array $B$ simulates the FIFO write buffers in a TSO (resp. PSO) execution, but buffers the pending store events rather than just the pending values to be written. Lines 16-25 update these write buffers, queueing a store when the SC execution performs a store and flushing the buffers when the SC execution performs an atomic operation.

But when should this simulated TSO (resp. PSO) execution "commit" these pending stores, making them globally visible? Lines 10-12 commit these pending stores as late as possible while still ensuring that the simulated TSO (resp. PSO) execution has the same trace and $\rightarrow_{h b}$-relation as the SC execution. This is achieved by, just before simulating operation $e$ by process $p$ on address $a$, committing all pending stores to address $a$ from any other process - these pending stores happen before $e$ in the SC execution, so they must be committed to ensure they happen before $e$ in the simulated TSO (resp. PSO) execution. Note that, in the TSO monitor, this may commit pending stores to other addresses, as well. 
1 B Proc $]$ : FIFOQueue of (Adr, Event)

$2 \operatorname{prev}[$ Proc $]$ : Event initialized to $\perp$

$\operatorname{monitor}_{T S O}(\mathrm{e})$ :

// Could simulation have $\rightarrow h b-$ cycle?

if $\exists\left(a(e), e^{\prime}\right) \in B\left[p\left(e^{\prime}\right)\right]$ with $p\left(e^{\prime}\right) \neq p(e) \wedge e^{\prime} \rightarrow_{h b}^{*} \operatorname{prev}[p(e)]:$

7

// Ensure equivalence to $S C$.

while $\exists(a(e), *) \in B\left[p^{\prime}\right]$ with $p^{\prime} \neq p(e):{ }_{10}$

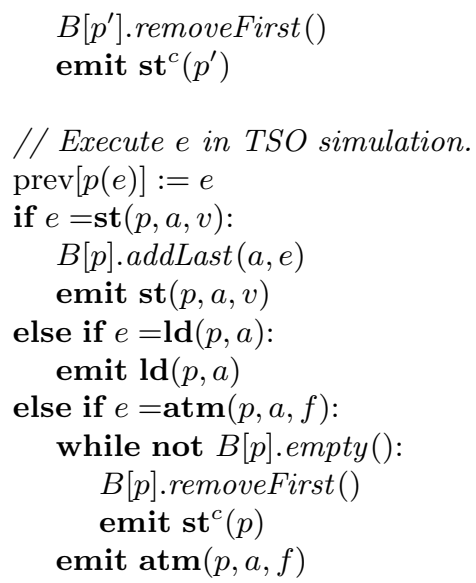

Fig. 4. Monitoring algorithm for TSO

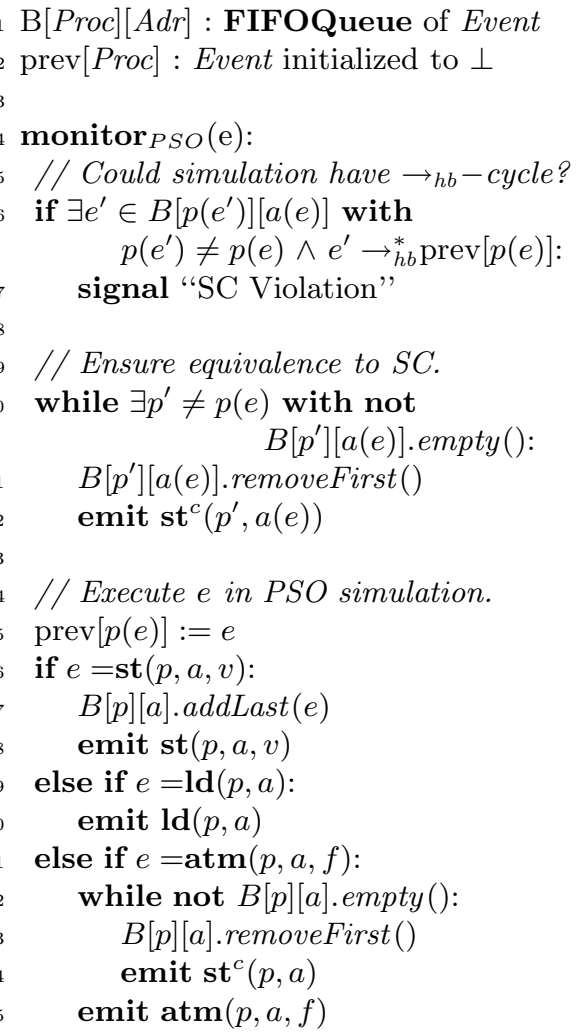

Fig. 5. Monitoring algorithm for PSO

But first, Line 6 of monitor $_{T S O}(e)$, resp. monitor ${ }_{P S O}(e)$, checks if we can create a violation of sequential consistency by executing memory operation $e$ in the TSO (resp. PSO) simulation before committing any pending and conflicting stores. That is, suppose $e$ is an operation on address $a$ by process $p$, and in our simulated TSO (resp. PSO) execution there is a pending store $e^{\prime}$ to $a$ by process $p^{\prime} \neq p$. In the TSO (resp. PSO) execution, we can force $e \rightarrow_{c} e^{\prime}$ by executing $e$ (and committing $e$, if it is a store) before committing $e^{\prime}$. Further, suppose that $e^{\prime}$ satisfies the rest of the condition at Line 6 . That is, $e^{\prime} \rightarrow{ }_{h b}^{*} \operatorname{prev}[p]$ - in the trace of the SC execution, event $e^{\prime}$ happens before the event $\operatorname{prev}[p]$ issued by process $p$ just before $e$. Then, as proved in Theorem 1 in the trace of the simulated TSO (resp. PSO) execution we will have $e^{\prime} \rightarrow_{h b}^{*} \operatorname{prev}[p] \rightarrow_{p} e \rightarrow_{c} e^{\prime}$. This is a cycle in the $\rightarrow_{h b}$-relation, indicating that the simulated TSO (resp. PSO) execution is not sequentially consistent.

In order to track the classic $\rightarrow_{h b}^{*}$ relation on the trace of the SC execution that we are monitoring, we use a well-known vector clock algorithm. The algorithm has a time complexity of $O(N \cdot P)$ on a trace/execution of length $N$ with $P$ processes. A short description of the algorithm can be found in, e.g., [11. 
Theorem 1. Algorithms monitor $_{T S O}$ and monitor $_{P S O}$ are sound monitoring algorithms for TSO and PSO, respectively. That is, whenever either reports a violation of sequential consistency given an $S C$ execution of a program $P$, then $P$ really has a violation of sequential consistency under TSO (resp. PSO).

Theorem 2. Algorithms monitor $_{T S O}$ and monitor $_{P S O}$ are complete for TSO and PSO, respectively. That is, if program $P$ has a violation of sequential consistency under TSO (resp. PSO), then there exists some $S C$ execution of $P$ on ${\text { which } \text { monitor }_{T S O} \text { (resp. monitor }}_{P S O}$ ) reports an $S C$ violation.

Theorem 3. On a sequentially consistent execution of length $N$ on $P$ processes, monitoring algorithms monitor $_{T S O}$ and monitor $_{P S O}$ run in time $O(N \cdot P)$. We sketch the proofs of these results in the Appendix. Complete proofs can be found in our accompanying technical report.

\section{Comparison to SOBER}

Our work is inspired by SOBER [4, a previous monitoring algorithm that detects program executions under TSO that violate sequential consistency by examining only SC executions. SOBER is derived from the axiomatic characterization of relaxed memory model TSO, while we work from operational definitions of TSO and PSO. There are four key differences between our work and SOBER.

First, we give monitor algorithms for detecting sequential consistency violations under both TSO and the more relaxed PSO memory model, while SOBER detects only violations under TSO.

Second, the run-time complexity of our algorithms is $O(N \cdot P)$, where $P$ is the number of processors and $N$ is the length of the monitored SC execution. This is an improvement over the complexity $O\left(N \cdot P^{2}\right)$ of the SOBER algorithm. The additional factor of $O(P)$ in SOBER is from a vector clock algorithm to maintain the relaxed happens-before relation, which axiomatically defines the behaviors legal under TSO. In contrast, when working from our operational definitions for TSO and PSO, there is no need for such additional vector clocks.

Third, the SOBER monitoring algorithm is more sensitive than our monitor $_{T S O}$. That is, for some programs there exist individual sequentially consistent executions for which SOBER will report the existence of an SC violation while monitor $_{T S O}$ will not. However, this does not affect the completeness of our monitoring algorithms - for such programs, there will always exist some other SC execution on which monitor $_{T S O}$ will detect and report a violation of sequential consistency. In our experimental evaluation, this reduced sensitivity does not seem to hinder our ability to find violations of sequential consistency when combining our monitors with preemption-bounded model checking [19].

Fourth, we believe that working with operational definitions for relaxed memory models TSO and PSO is both simpler than working with axiomatic definitions and leads to more natural and intuitive monitoring algorithms. As evidence for this belief, we note that we have discovered [8] a subtle error in the axiomatic definition of TSO given in 4, which leads SOBER to fail to detect some real violations of sequential consistency under TSO. This error has been confirmed [7] 
both by the authors of [4] and by [18]. We discuss this error, and how to correct it, in our accompanying technical report.

\section{Experimental Evaluation}

In order to experimentally evaluate our monitor algorithms, we have implemented monitor $_{T S O}$ and monitor $_{P S O}$ on top of the THRILLE [16] tool for model checking, testing, and debugging parallel $\mathrm{C}$ programs.

In our experiments, we use seven benchmarks. The names and sizes of these benchmarks are given in Columns 1 and 2 of Tables 1 and 2. Five are implementations of concurrent data structures taken from [3]: msn, a non-blocking queue, ms2, a two-lock queue, lazylist, a list-based concurrent set, harris, a non-blocking set, and snark, a double-ended queue (dequeue). The other two benchmarks are implementations of Dekker's algorithm and Lamport's bakery algorithm for mutual exclusion. Previous research 34 has demonstrated that the benchmarks have sequential consistency violations under relaxed memory models without added memory fences. For each of the benchmarks we have manually constructed a test harness.

In our experimental evaluation, we combine our monitoring algorithms with THRILLE's preemption-bounded [19 model checking. That is, we run monitor $_{T S O}$ and monitor $P S O$ on all sequentially consistent executions of each benchmark with a bounded number of preemptive context switches. This verification is not complete-because we do not apply our monitor algorithms to every SC execution, we may miss possible violations of SC under TSO or PSO. We evaluate only whether our monitoring algorithms are effective in finding real violations of $\mathrm{SC}$ when combined with a systematic but incomplete model checker.

We run two sets of experiments, one with a preemption bound of 1 and the other with a preemption bound of 2 . Columns 3 and 4 list the number of parallel interleavings explored and the total time taken with a preemption bound of 1 (Table1) and a bound of 2 (Table 2). The cost of running our unoptimized monitor implementations on every sequentially consistent execution adds an overhead of roughly $20 \%$ to THRILLE's model checking for the data structure benchmarks and about $100 \%$ to the mutual exclusion benchmarks.

Table 1. Experimental evaluation of $\operatorname{monitor}_{T S O}$ and monitor $_{P S O}$ on all interleavings with up to 1 preemption

\begin{tabular}{|c|c|c|c|c|c|}
\hline bench & LOC & $\begin{array}{c}\text { \# inter- } \\
\text { leavings }\end{array}$ & $\begin{array}{c}\text { total } \\
\text { time }\end{array}$ & $\begin{array}{c}\text { distinct violations } \\
\text { under TSO }\end{array}$ & $\begin{array}{c}\text { distinct violations } \\
\text { under PSO }\end{array}$ \\
\hline dekker & 20 & 79 & 6.4 & 3 & 5 \\
\hline bakery & 30 & 197 & 42.4 & 3 & 4 \\
\hline msn & 80 & 92 & 7.5 & 0 & 3 \\
\hline ms2 & 80 & 123 & 11.0 & 0 & 2 \\
\hline harris & 160 & 161 & 18.8 & 0 & 4 \\
\hline lazylist & 120 & 139 & 14.0 & 0 & 4 \\
\hline snark & 150 & 172 & 15.4 & 0 & \\
\hline
\end{tabular}


Table 2. Experimental evaluation on all interleavings with up to 2 preemptions

\begin{tabular}{|c|c|c|c|c|c|}
\hline bench & LOC & $\begin{array}{c}\text { \# inter- } \\
\text { leavings }\end{array}$ & $\begin{array}{c}\text { total } \\
\text { time }\end{array}$ & $\begin{array}{c}\text { distinct violations } \\
\text { under TSO }\end{array}$ & $\begin{array}{c}\text { distinct violations } \\
\text { under PSO }\end{array}$ \\
\hline dekker & 20 & 1714 & 180.0 & 9 & 11 \\
\hline bakery & 30 & 13632 & 3992.4 & 3 & 4 \\
\hline msn & 80 & 2300 & 196.1 & 0 & 3 \\
\hline ms2 & 80 & 3322 & 300.0 & 0 & 2 \\
\hline harris & 160 & 5646 & 661.7 & 0 & 4 \\
\hline lazylist & 120 & 4045 & 428.4 & 0 & 10 \\
\hline snark & 150 & 6510 & 609.9 & 0 & 2 \\
\hline
\end{tabular}

Rather than report every single parallel interleaving on which one of our monitor algorithms signaled a violation, we group together violations caused by the same pair of operations $e$ and $e^{\prime}$. We say that a violation is caused by $e$ and $e^{\prime}$ when monitor $_{T S O}(e)$ or $\operatorname{monitor}_{P S O}(e)$ is the call on which a violation is signaled, and $e^{\prime}$ is the conflicting memory access identified in the condition at Line 6 . For such a violation, $e^{\prime}$ happens before the event $\operatorname{prev}[p(e)]$ just before $e$ in process $p(e)$, but event $e$ also happens before $e^{\prime}$ because we delay store $e^{\prime}$ until after $e$ completes in the violating TSO or PSO execution.

Columns 4 and 5 of Tables 1 and 2 list the number of such distinct violations of sequential consistency found under TSO and PSO in our experiments. Note that we find no violations of sequential consistency under TSO for any of the data structure benchmarks. Their use of locks and compare-and-swap operations appear to be sufficient to ensure sequential consistency under TSO. On the other hand, we find violations of sequential consistency for all benchmarks under PSO.

Acknowledgments. We would like to thank Krste Asanović, Pallavi Joshi, ChangSeo Park, and our anonymous reviewers for their valuable comments. This research supported in part by Microsoft (Award \#024263) and Intel (Award \#024894) funding and by matching funding by U.C. Discovery (Award \#DIG07-10227), by NSF Grants CNS-0720906, CCF-1018729, and CCF-1018730, and by a DoD NDSEG Graduate Fellowship. Additional support comes from Par Lab affiliates National Instruments, NEC, Nokia, NVIDIA, Samsung, and Sun Microsystems.

\section{References}

1. Arvind, A., Maessen, J.W.: Memory model = instruction reordering + store atomicity. In: ISCA 2006: Proceedings of the 33rd Annual International Symposium on Computer Architecture, pp. 29-40. IEEE Computer Society, Los Alamitos (2006)

2. Atig, M.F., Bouajjani, A., Burckhardt, S., Musuvathi, M.: On the verification problem for weak memory models. In: The 36th Annual ACM SIGPLAN-SIGACT Symposium on Principles of Programming Languages, POPL (2010)

3. Burckhardt, S., Alur, R., Martin, M.M.K.: CheckFence: checking consistency of concurrent data types on relaxed memory models. In: ACM SIGPLAN Conference on Programming Language Design and Implementation (2007) 
4. Burckhardt, S., Musuvathi, M.: Effective program verification for relaxed memory models. In: Gupta, A., Malik, S. (eds.) CAV 2008. LNCS, vol. 5123, pp. 107-120. Springer, Heidelberg (2008)

5. Burckhardt, S., Musuvathi, M.: Effective program verification for relaxed memory models. Tech. Rep. MSR-TR-2008-12, Microsoft Research (2008)

6. Burckhardt, S., Musuvathi, M.: Memory model safety of programs. In (EC) ${ }^{2}$ : Workshop on Exploting Concurrency Efficiently and Correctly (2008)

7. Burckhardt, S., Musuvathi, M.: Personal communcation (2010)

8. Burnim, J., Sen, K., Stergiou, C.: Sound and complete monitoring of sequential consistency in relaxed memory models. Tech. Rep. UCB/EECS-2010-31, EECS Department, University of California, Berkeley (March 2010), http://www . eecs.berkeley.edu/Pubs/TechRpts/2010/EECS-2010-31.html

9. Dill, D.L., Park, S., Nowatzyk, A.G.: Formal specification of abstract memory models. In: Symposium on Research on Integrated Systems (1993)

10. Flanagan, C., Freund, S.N.: Adversarial memory for detecting destructive races. In: ACM SIGPLAN Conference on Programming Language Design and Implementation, PLDI (2010)

11. Flanagan, C., Godefroid, P.: Dynamic partial-order reduction for model checking software. In: Proc. of the 32nd Symposium on Principles of Programming Languages (POPL 2005), pp. 110-121 (2005)

12. Gibbons, P., Korach, E.: The complexity of sequential consistency. In: Fourth IEEE Symposium on Parallel and Distributed Processing. pp. 317-235 (1992)

13. Gopalakrishnan, G., Yang, Y., Sivaraj, H.: QB or not QB: An efficient execution verification tool for memory orderings. In: Alur, R., Peled, D.A. (eds.) CAV 2004. LNCS, vol. 3114, pp. 401-413. Springer, Heidelberg (2004)

14. Higham, L., Kawash, J., Verwaal, N.: Weak memory consistency models. part i: Definitions and comparisons. Tech. Rep. 97/603/05, Department of Computer Science, The University of Calgary (1998)

15. Huynh, T.Q., Roychoudhury, A.: Memory model sensitive bytecode verification. FMSD 31(3), 281-305 (2007)

16. Jalbert, N., Sen, K.: A trace simplification technique for effective debugging of concurrent programs. In: The 18th ACM SIGSOFT International Symposium on the Foundations of Software Engineering (SIGSOFT 2010/FSE-18) (2010)

17. Lamport, L.: How to make a multiprocessor computer that correctly executes multiprocess programs. IEEE Trans. Comput. 28(9), 690-691 (1979)

18. Mador-Haim, S., Alur, R., Martin, M.M.: Generating litmus tests for contrasting memory consistency models. In: Touili, T., Cook, B., Jackson, P. (eds.) CAV 2010. LNCS, vol. 6174, pp. 273-287. Springer, Heidelberg (2010)

19. Musuvathi, M., Qadeer, S.: Iterative context bounding for systematic testing of multithreaded programs. In: PLDI 2007: Proceedings of the 2007 ACM SIGPLAN Conference on Programming Language Design and Implementation. ACM, New York (2007)

20. Park, S., Dill, D.L.: An executable specification, analyzer and verifier for RMO (relaxed memory order). In: ACM Symposium on Parallel Algorithms and Achitectures, pp. 34-41. ACM Press, New York (1995)

21. Sewell, P., Sarkar, S., Owens, S., Nardelli, F.Z., Myreen, M.O.: x86-TSO: a rigorous and usable programmer's model for x86 multiprocessors. Commun. ACM 53(7), 89-97 (2010)

22. Shasha, D., Snir, M.: Efficient and correct execution of parallel programs that share memory. ACM Trans. Program. Lang. Syst. 10(2), 282-312 (1988)

23. SPARC International. The SPARC architecture manual (v. 9). Prentice-Hall, Englewood Cliffs (1994) 


\section{A Soundness and Completeness Proof Sketches}

We sketch here proofs of the soundness and completeness of our monitoring algorithms. Complete proofs can be found in our accompanying technical report.

Theorem 1. Algorithms monitor $_{T S O}$ and monitor $_{P S O}$ are sound monitoring algorithms for TSO and PSO, respectively.

Proof (sketch). Let $\left(\sigma_{0}, m_{0}\right) \stackrel{e_{1}, r_{1}}{\longrightarrow} \cdots \stackrel{e_{n}, r_{n}}{\longrightarrow}\left(\sigma_{n}, m_{n}\right)$ be an SC execution of a program $P$ such that monitor $_{T S O}$ (resp., monitor ${ }_{P S O}$ ), on this execution, first signals "Sequential Consistency Violation" on event $e_{n}$. We prove that $P$ has an execution under TSO (resp., PSO) with a non-sequentially-consistent trace.

Observe that, during its execution, monitor mSO $_{T S}$ (resp., monitor $_{P S O}$ ) emits labels from Event and $\tau_{T S O}$ (resp., $\left.\tau_{P S O}\right)$. We can show:

(1) That the events emitted during the first $n-1$ calls to monitor mSO $_{P S}$ (resp., monitor $_{P S O}$ ) form a TSO (resp., PSO) execution of program $P$. Further, this execution has the same trace as the SC execution.

We can show that the emitted $\mathbf{s t}^{c}$ transitions ensure that the loads and atomic operations in the TSO (resp., PSO) execution see the same values as in the SC execution. Thus, program $P$ behaves identically.

(2) That operation $e_{n}$ can be performed in this TSO (resp., PSO) execution in a way that creates a trace with a cycle in its $\rightarrow h b$-relation.

In the emitted TSO (resp., PSO) execution, the event $e^{\prime}$ in the condition at Line 6 is still pending. Thus, we can perform $e$ (and commit it, if it is a store) before $e^{\prime}$ commits, so that $e \rightarrow_{c} e^{\prime}$. And $e^{\prime} \rightarrow_{h b}^{*} \operatorname{prev}[p(e)]$ because the emitted execution has the same trace as the $\mathrm{SC}$ execution $e_{1}, \ldots, e_{n-1}$. Thus, we create a TSO (resp., PSO) execution with happens-before cycle:

$$
e^{\prime} \rightarrow_{h b}^{*} \operatorname{prev}[p(e)] \rightarrow_{p} e \rightarrow_{c} e^{\prime}
$$

Theorem 2. Algorithms monitor $_{T S O}$ and monitor $_{P S O}$ are complete monitoring algorithms for TSO and PSO, respectively.

Proof (sketch). Suppose $\left(s_{0}, m_{0}\right) \stackrel{l_{1}}{\longrightarrow} \cdots \stackrel{l_{n}}{\longrightarrow}\left(s_{n}, m_{n}\right)$ is a TSO (resp., PSO) execution of program $P$ with a trace $\left(E, \rightarrow_{p}, s r c, \rightarrow_{s t}\right)$ that is not sequentially consistent. Recall that each $l_{i}$ is either a memory event from Event or an internal transition $\mathbf{s t}^{c}(p)$ for TSO or $\mathbf{s t}^{c}(p, a)$ for PSO.

We can obtain shorter TSO (resp., PSO) executions of $P$ by removing some Event-labeled transition from the execution $l_{1}, \ldots, l_{n}$, as well as possibly removing corresponding $\mathbf{s t}^{c}$ transitions. For example, we can safely remove the last Event issued by any process $p$, even if it is not last in the execution, as long as it does not write a value that is read by a later operation.

We use this freedom to construct a shorter TSO (resp., PSO) execution that is a minimal violation of sequential consistency. That is, if any further Event's are removed, the trace of the execution becomes sequentially consistent.

On this minimally-violating execution, consider the Event's that can be safely removed - i.e. their removal leaves a valid TSO (resp., PSO) execution, but this 
execution has an SC trace. For such a safely-removable $e$, let last(e) denote the last write to $a(e)$ to become globally visible in the TSO (resp., PSO) execution, not including $e$ itself. In the TSO (resp., PSO) execution $e \rightarrow_{c}$ last, but we would have last $(e) \rightarrow_{h b}^{*} e$ in the $\mathrm{SC}$ execution in which $e$ is removed and then run at the end. We can show that, for at least one of the these $e$, no other event comes after last $(e)$ in any $\mathrm{SC}$ trace and also forces last $(e)$ to be committed before event $e$ executes. Thus, monitor mSO/PSO $_{\text {(e) }}$ reports a violation on this execution.

\section{B Complexity of Monitoring Algorithms}

Lemma 1. During the execution of monitor $_{T S O}$ (respectively, monitor $\left._{P S O}\right)$, for each address $a \in A d r$, at any given time at most one process $p \in$ Proc will have pending stores to address $a$ in its write buffer $B[p]$ (resp., $B[p][a]$ ).

Proof (by induction). Initially, before any calls to monitor $\mathbf{m}_{T S O}$ (resp., monitor $\left._{P S O}\right)$, the lemma clearly holds.

Suppose the lemma holds after $k$ calls to monitor $_{T S O}$ (resp., monitor $_{P S O}$ ), and let $\operatorname{monitor}_{T S O}(e)$ or $\operatorname{monitor}_{P S O}(e)$ be the $(k+1)$-st call. If $e$ is not a store, or if $e=\operatorname{st}(p, a, v)$ where no other process $p^{\prime} \neq p$ has any pending stores to $a$, then the lemma clearly holds during and after the call.

Suppose instead that $e=\mathbf{s t}(p, a, v)$ and process $p^{\prime} \neq p$ is the only process with pending stores to address $a$. Then, in monitor $_{T S O}$ (resp., monitor m $\left._{P O}\right)$, the while-loop at Lines 10-12 commits all such pending stores by $p^{\prime}$ before Line 17 adds a pending store to $a$ to a write buffer of process $p$.

By Lemma 1, in the condition at Line 6 in $\operatorname{monitor}_{T S O}(e)$ and $\operatorname{monitor}_{P S O}(e)$ at most one processor $p^{\prime}$ can have pending writes to $a(e)$.

We further observe that monitor $_{T S O}$ and monitor $_{P S O}$ remain complete if, at Line 6 , we check this condition with only the last (i.e. most recent) pending store to $a$ in $B\left[p^{\prime}\right]$ or $B\left[p^{\prime}\right][a]$. See the proof of Theorem 2 for details.

Theorem 3. On a sequentially consistent execution of length $N$ on $P$ processes,

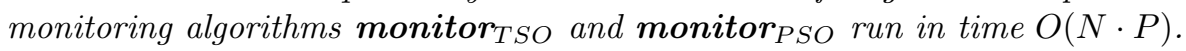

Proof. We show that each call monitor $_{T S O / P S O}(e)$ runs in amortized $O(P)$ time, yielding $O(N \cdot P)$ total time. As mentioned in the previous section, updating the vector clocks to maintain the happens-before relation on the monitored $\mathrm{SC}$ execution requires $O(P)$ time per call to monitor $_{T S O / P S O}(e)$.

For each address $a \in A d r$, we track the single process $p$ which has pending stores to $a$. Further, for $a$ we maintain a pointer into the FIFOQueue of this process to the last (i.e. most recent) pending store to $a$. We can maintain these two pieces of per-address information in $O(1)$ time per call to monitor $_{T S O / P S O}(e)$. Using this information, checking the condition at Line 6 requires $O(1)$ time to find the last pending store to $a(e)$ and $O(1)$ time to check $e^{\prime} \rightarrow_{h b}^{*} e$. The condition at Line 10 can similarly be checked in $O(1)$.

Finally, the total number of iterations of the while-loops at Lines 10 and 22, across all $N$ calls to monitor $_{T S O / P S O}(e)$, cannot exceed $O(N)$ as we buffer no more than $N$ writes. 